\title{
Method of Safe Returning of the Vessel to Planned Route After Deviation from Collision
}

\author{
M. Tsymbal \& I. Urbansky \\ Odessa National Maritime Academy, Odessa, Ukraine
}

\begin{abstract}
Flexible strategies for collision avoidance, presented at TransNav 2007, were examined using computer program for its correctness in different situations of ships interaction. It was determined, that on short distance the risk of collision can arise again when the vessel returning to the planned route after deviation from collision. For controlling ship's safe returning, the mathematical model was developed. This model describes the analytical dependence of the rate of changing relative course with respect to rates of turning of the vessels and its initial relative position. This method can be used in automatic systems for controlling the safe returning of the vessel to the planned route.
\end{abstract}

\section{INTRODUCTION}

Flexible strategies for collision avoidance, take into account the presence of dangerous and obstacle vessels, hazards for navigation and the Colreg requirements. The strategy of collision avoidance depends on the realized range of mutual duties, relative position of vessel and target and, correlation of their speeds. In general strategy of deviation foresees transfer the current position of the vessel from subset of dangerous positions to subset of safe positions, calculation the deviation course and then returning to the planned route by a course tangent to the circle of the assigned CPA (Tsymbal 2006, 2007, 2008).

During modeling the flexible strategies on computer, it was determined, that on short distances the risk of collision can arise again when the vessel returning to the planned route after deviation from collision.

The paper presents the method for calculating the parameters for ship manoeuvring, when returning to the planned route after deviation from collision.

\section{MATHEMATICAL MODEL}

\subsection{Three types of returning trajectories}

The detailed analysis, shows that for safe returning of the vessel on the planned route in default of coordination between the ship and target it is necessary the initial situation $G$ to identify with one of three subsets Mn1, Mn2 or Mn3, each of which determines the type of trajectory for returning the ship and mathematical model for calculation the parameters of manoeuvre.

The first subset $M_{n 1}$ includes safe situations $G$, when are assured increase the distance $L_{t}$ between the vessels and target, i.e. $d L_{t} / d t>0$. Second subset $M_{n 2}$ includes situations, when distance between the ship and target reduces, i.e. $d L_{t} / d t<0$. And, finally, the third subset of situations $M_{n 3}$ includes those situations, for which it is possible increasing or reducing the distance $L_{t}$.

For identification the type of initial situation $G$ it is necessary to calculate the initial relative course $K_{\text {oto }}$ and rate of its change $\omega_{o t b}$. 


\subsection{Calculation of relative course and rate of its change}

The initial relative course $K_{\text {oto }}$ means the relative course of the ship's deviation. Its calculation is produced on the parameters of motion of the vessel and target by expression:

$K_{\text {oto }}=\arcsin \left[\frac{V_{v} \sin K_{v o}-V_{c} \sin K_{c o}}{V_{\text {oto }}}\right]$

where $K_{v o}, V_{v}, K_{c o}$, and $V_{c}$, $=$ the values of initial course and speed of the vessel and target accordingly; $V_{\text {oto }}=$ initial relative speed:

$V_{\text {oto }}=\left[V_{v}^{2}+V_{c}^{2}-2 V_{v} V_{c} \cos \left(K_{v o}-K_{c o}\right)\right]^{1 / 2}$

For calculation the value of relative angular speed $\omega_{\text {otb }}$, it is necessary to know the values of rate of turn of the vessel $\omega_{v b}$ and the target $\omega_{c}$.

Calculation of the value relative angular speed $\omega_{\text {otb }}$ for a situation, when the vessel and target change course simultaneously is produced by the following analytical expression:

$\omega_{o t b}=\frac{V_{v} \cos \left(K_{v t}\right) \omega_{v b}-V_{c} \cos \left(K_{c t}\right) \omega_{c}}{V_{v} \cos \left(K_{v t}\right)+V_{c} \cos \left(K_{c t}\right)}-$

$\left[V_{v} \sin \left(K_{v t}\right)-V_{c} \sin \left(K_{c t}\right)\right] V_{v} V_{c} \sin \left(\Delta K_{y}+\Delta \omega_{b} t\right) \Delta \omega_{b}$

$\left[V_{v} \cos \left(K_{v t}\right)+V_{c} \cos \left(K_{c t}\right)\right] V_{o t}^{2}$

where $K_{v t}=\left(K_{v y}+\omega_{y b} t\right) ; \quad K_{c t}=\left(K_{v y}+\omega_{y b} t\right)$;

$\Delta \omega_{b}=\omega_{y b}-\omega_{c} ; \quad \Delta K=K_{v y}-K_{c y} ; \quad K_{v y}=$ vessel's course deviation; $K_{c y}=$ target's course deviation. $K_{v y}$ and $K_{c y}$ determined previously by using the method of flexible strategies for collision avoidance.

When target keep her course, $\omega_{c}=0$ and, only the vessel change her course

$$
\begin{aligned}
& \omega_{o t b}=\frac{V_{v} \cos \left(K_{v t}\right) \omega_{v b}}{V_{v} \cos \left(K_{v t}\right)+V_{c} \cos \left(K_{c y}\right)}- \\
& -\frac{\left[V_{v} \sin \left(K_{v t}\right)-V_{c} \sin \left(K_{c y}\right)\right] V_{v} V_{c} \sin \left(\Delta K_{y}+\omega_{y b} t\right) \omega_{y b}}{\left[V_{v} \cos \left(K_{v t}\right)+V_{c} \cos \left(K_{c y}\right)\right] V_{o t}{ }^{2}}
\end{aligned}
$$

The sign of the vessel's rate of turn depends on the side of turn $\Delta_{y}$, so, that $\operatorname{sign}\left(\omega_{y b}\right)=-\operatorname{sign}\left(\Delta_{y}\right)$; $\Delta_{y}=1$ when deviation to starboard.

\subsection{Identification of initial situation}

Belonging of the initial situation $\mathrm{G}$ to the subset $\mathrm{M}_{\mathrm{n}}$ is analytically expressed as follows:

$$
\begin{aligned}
& G \in M n_{1}, \text { if }\left\{K_{o t o} \in\left[\alpha_{o}+\pi / 2, \alpha_{o}+\pi\right] \text { and } \omega_{o t b}>0\right\}, \\
& \text { or }\left\{K_{o t o} \in\left[\alpha_{o}+\pi, \alpha_{o}-\pi / 2\right] \text { and } \omega_{o t b<0\}} ;\right. \\
& \operatorname{sign}\left(\omega_{V b}\right)=-\operatorname{sign}\left(\Delta_{y}\right)
\end{aligned}
$$

where $\alpha_{0}=$ initial bearing from the vessel to the target.

Condition which describes belonging of initial situation $G$ to the subset $M_{n 2}$, expressed by a next correlation of relative initial course $K_{\text {oto }}$ and sign of rate of turn $\omega_{o t b}$ :

$$
\begin{aligned}
& G \in M n_{2}, \text { if }\left\{K_{o t o} \in\left[\alpha_{o}-\pi / 2, \alpha_{o}\right] \text { and } \omega_{o t b}>0\right\}, \text { or } \\
& \left\{K_{o t o} \in\left[\alpha_{o}, \alpha_{o}+\pi / 2\right] \text { and } \omega_{o t b<0\} ;}\right. \\
& \operatorname{sign}\left(\omega_{V b}\right)=-\operatorname{sign}\left(\Delta_{y}\right)
\end{aligned}
$$

Belonging of initial situation $G$ to subset $M_{n 3}$, is determined by analytical expressions:

$$
\begin{aligned}
& G \in M n_{3} \text {, if }\left\{K_{o t o} \in\left[\alpha_{o}-\pi / 2, \alpha_{o}\right] \text { and } \omega_{o t b}<0\right\} \text {, or } \\
& \left\{K_{o t o} \in\left[\alpha_{o}, \alpha_{o}+\pi / 2\right] \text { and } \omega_{o t b}>0\right\} \text {, or } \\
& \left\{K_{o t o} \in\left[\alpha_{o}+\pi / 2, \alpha_{o}+\pi\right] \text { and } \omega_{o t b<0}\right\} \text {,or } \\
& \left\{K_{o t o} \in\left[\alpha_{o}+\pi, \alpha_{o}-\pi / 2\right] \text { and } \omega_{o t b}>0\right\} \text {; } \\
& \operatorname{sign}\left(\omega_{V b}\right)=-\operatorname{sign}\left(\Delta_{y}\right)
\end{aligned}
$$

The type of returning trajectory depends on the subset to which the initial situation belongs.

\subsection{First type of returning trajectory}

If $\mathrm{G}$ belonging to $\mathrm{Mn} 1$, the most preferable is the first type of vessel's returning trajectory to the planned route. This type of trajectory requires minimum time. The first type trajectory is shown on Figure 1.

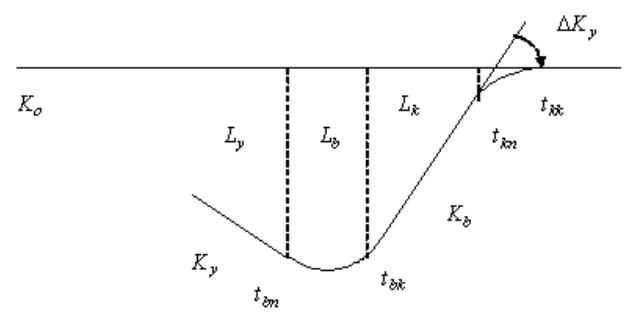

Figure 1. First type of returning trajectory in true motion

This manoeuvre includes the turn of vessel from the course of deviation $K_{y}$ to the returning course $K_{b}$, and, when approaching to the planned route the vessel shall turn from the course $K_{b}$ on a programmatic course $K_{0}$, as shown on a Fig. 1 in true motion. The value of $K_{b}$ depends on $K_{0}$ and $\Delta K_{y}$. We proposed $\Delta K_{y}=40^{\circ}$.

The parameters of this manoeuvre are the values of: returning course $K_{b}$; the moments of beginning the turn $t_{b n}$ and ending the first turn $t_{b k}$; the moments $t_{k n}$ and $t_{k k}-$ which determine the beginning end ending of the second turn. These parameters calculated by next equations:

$$
L_{k}=R_{c}\left(1-\cos \Delta K_{y}\right)
$$


where $R_{c}=$ radius of circulation of the vessel.

$\Delta t_{b n}=2 \Delta K_{y} / \omega_{V}$

$t_{b k}=t_{b n}+\Delta t_{b n}$

$L_{b}=\frac{\left(L_{y}-L_{k}\right)}{\sin \Delta K_{y}}$

$t_{k n}=t_{b k}+\frac{\left(L_{y}-L_{k}\right)}{V_{v} \sin \Delta K_{y}}$

$t_{k k}=t_{k n}+\Delta K_{y} / \omega_{V}$

\subsection{Second type of returning trajectory}

In case when $G$ belonging to $M_{n 2}$, the vessel use second type of returning trajectory which consist with the first turn to the same side as deviation from collision with angular speed $\omega_{y b}=-\operatorname{sign}\left(\Delta_{y}\right) \omega_{\mathrm{ymax}}$ and, then returning to the planned route by course $K_{b}$. The second type of trajectory is shown on Figure 2 in true motion.

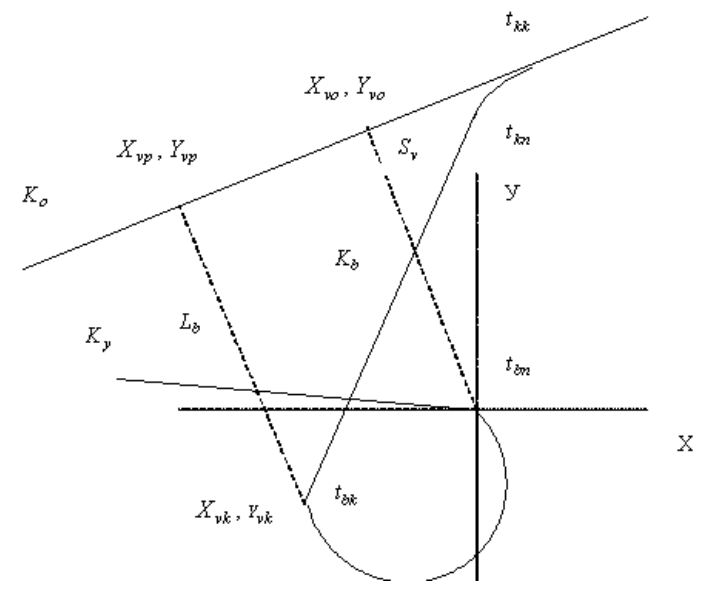

Figure 2. Second type of returning trajectory in true motion

On the first step it is necessary to determine the coordinates $X_{v o}, Y_{v o} X_{v k} Y_{v k} X_{v p} Y_{v p}$ :

$X_{v o}=-S_{v} \cos K_{o}$

$Y_{v o}=S_{v} \sin K_{o}$

$X_{v k}=R_{c}\left[\cos K_{y}-\cos \left(K_{y}-K_{b}\right)\right]$

$Y_{v k}=R_{c}\left[\sin \left(K_{y}-K_{b}\right)-\sin K_{y}\right]$

$X_{v p}=\frac{\cos K_{o}\left[X_{v o} \cos K_{o}+\left(Y_{v k}-Y_{v o}\right) \sin K_{o}\right]}{\left(\cos ^{2} K_{o}-\sin ^{2} K_{o}\right)}$

$Y_{v p}=\left[Y_{v o} \sin K_{o}+\left(X_{v p}-X_{v o}\right) \cos K_{o}\right] / \sin K_{o}$

Then we calculate the distance $L_{b}$ :

$$
L_{b}=\sqrt{\left(X_{v p}-X_{v k}\right)^{2}+\left(Y_{v p}-Y_{v k}\right)^{2}}
$$

The parameters of manoeuvre calculated by next equations:

$$
\begin{aligned}
& t_{b k}=t_{b n}+\left[2 \pi-\left(K_{y}-K_{b}\right)\right] / \omega_{V} \\
& t_{k n}=t_{b k}+\frac{\left(L_{b}-L_{k}\right)}{V_{v} \sin \Delta K_{y}} \\
& t_{k k}=t_{k n}+\left(K_{b}-K_{o}\right) / \omega_{V}
\end{aligned}
$$

\subsection{Third type of returning trajectory}

If $G$ belonging to $M_{n 3}$, the vessel shall continue motion with relative course of deviation $K_{\text {oty }}$ till the moment, when the returning to relative course $K_{\text {otb }}$ guarantee that CPA will not less then $L_{d}$. This third type of returning trajectory shown on Figure 3 in relative motion.

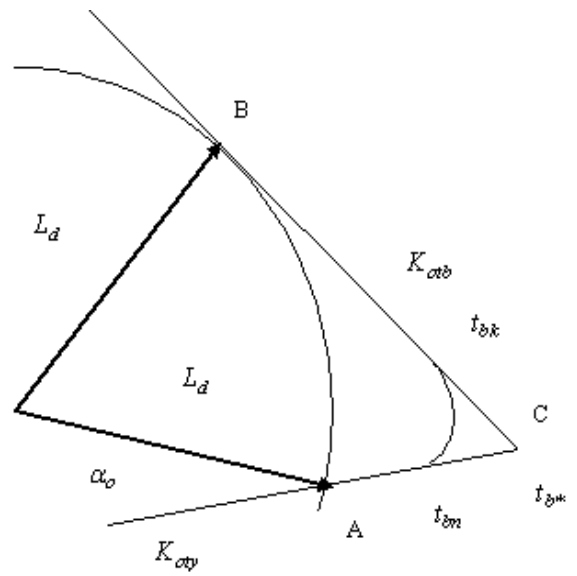

Figure 3. Third type of returning trajectory in relative motion

In the beginning it is necessary to calculate the co-ordinates of points A,B,C:

$X_{A}=L_{d} \sin \alpha_{o}$

$Y_{A}=L_{d} \cos \alpha_{o}$

$X_{B}=L_{d} \cos K_{o t b}$

$Y_{B}=-L_{d} \sin K_{o t b}$

where $K_{\text {otb }}=$ relative course of returning.

$$
\begin{aligned}
& Y_{C}=\frac{G}{\left(\operatorname{tg} K_{o t y}-\operatorname{tg} K_{o t b}\right)} \\
& X_{C}=X_{B}+\left[\frac{G}{\left(\operatorname{tg} K_{o t y}-\operatorname{tg} K_{o t b}\right)}-Y_{B}\right] \operatorname{tg} K_{o t b}
\end{aligned}
$$

where

$G=X_{B}-X_{A}+Y_{A} \operatorname{tg} K_{o t y}-Y_{B} \operatorname{tg} K_{o t b}$

$K_{\text {oty }}=$ relative course of deviation .

Distance $L_{A C}$ between points A and C calculated as follows:

$$
L_{A C}=\sqrt{\left(X_{C}-X_{A}\right)^{2}+\left(Y_{C}-Y_{A}\right)^{2}} .
$$


The moment of time to turn $t_{b^{*}}$ is determined by the following formula:

$t_{b^{*}}=\frac{L_{A C}}{V_{o t y}}=\frac{\sqrt{\left(X_{C}-X_{A}\right)^{2}+\left(Y_{C}-Y_{A}\right)^{2}}}{V_{o t y}}$

Amendment for ship's dynamic $\Delta t_{\mathrm{b}}$ is calculated on a formula:

$\Delta t_{b}=\left\{\sin K_{o t b} R_{c}\left(\sin K_{b}-\sin K_{y}\right)-\right.$

$\left.\cos K_{o t b} R_{c}\left(\cos K_{y}-\cos K_{b}\right)+V_{c} \tau \sin \left(K_{o t b}-K_{c}\right)\right\} /$

$\left[V_{o t y} \sin \left(K_{o t y}-K_{o t b}\right)\right]$

where $\tau=\frac{A b s\left(K_{b}-K_{y}\right)}{\omega_{v b}}$.

The parameters of beginning and ending the turn to course $K_{b}$ are calculated by next equations:

$t_{b n}=t_{b^{*}}-\Delta t_{b}$

$t_{b k}=t_{b n}+\tau$

\section{EXPERIMENTS AND RESULTS}

For verification of correctness theoretical results the imitation software was designed for modeling the manoeuvre of returning the vessel to the programmatic trajectory of motion in different situations and with different types of returning trajectory. This computer program allows on the set initial situation choose the safe manoeuvre of returning and builds his trajectory on the screen. Information appears in relative and true motion.

On Figure 4 a situation is shown, when the safe manoeuvre of returning is possible by the trajectory of the first type.

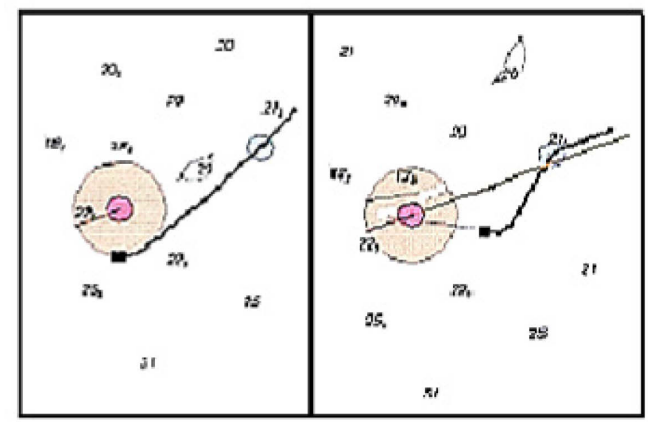

Figure 4. First type trajectory for safe returning

Left part of screen shows the relative trajectory of the vessel in relation to the immobile target. Right part of the screen contains the trajectories of the vessel and target in true motion. As we can see, on relative motion, the distance between vessel and target is increasing. Black square shows the initial position of the vessel after deviation from collision.
On Figure 5 second type of safe returning trajectory shown.

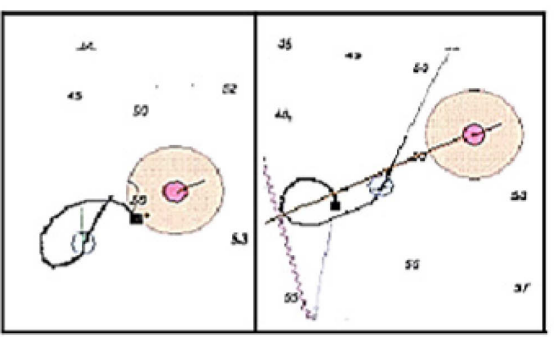

Figure 5. Second type trajectory for safe returning

Third type of safe returning trajectory shown on Figure 6.

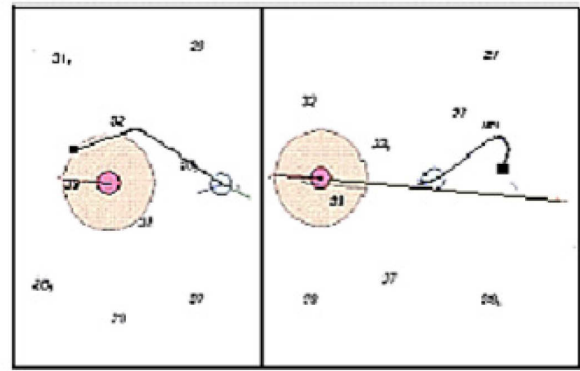

Figure 6. Third type of safe returning trajectory

More than 100 different initial situations were generated and the parameters of manoeuvres of returning on the programmatic trajectory of motion are calculated and modelling. It appeared that $51 \%$ of manoeuvres had the first type of trajectory of returning, $37 \%$ is the second type and $12 \%$ is the third type. All manoeuvres chosen by the program were safe.

\section{CONCLUSION}

This paper presented the method which taking into account high level of vagueness of target's conduct, and increase safe returning of the vessel to planned route after deviation from collision.

Obviously, that at presence of co-ordination between a vessel and target on the stage of their returning to planned route provides more high safety returning.

\section{REFERENCES}

Tsymbal M., Burmaka I. \& Tjupikov E. 2007, Flexible strategies for preventing collisions. Monograph, Odessa, Ukraine, 2007, ISBN 978-966-8128-96-7

Tsymbal M., 2007. Method of Synthesis of Flexible Strategies for Preventing Collisions. In Weintrit, A. (Ed.): Advances in Marine Navigation and Safety of Transportation. Monograph, Gdynia, June 2007, ISBN 978-83-7421-018-8

Tsymbal M. \& Urbansky I. 2008. Development of simulator systems for preventing collision of ships. In Benedict $\mathrm{K}$. (Ed.): 35th Annual General Meeting and International Workshop Conference in Intemational Marine Simulator Forum, Rostok, September 2008, ISBN 978-3-939159-55-1 